Med Klin Intensivmed Notfmed 2020 115:486-487 https://doi.org/10.1007/s00063-020-00704-0 Online publiziert: 25 . Juni 2020

(c) Springer Medizin Verlag GmbH, ein Teil von Springer Nature 2020

\section{Guido Michels ${ }^{1} \cdot$ Uta Ochmann ${ }^{2} \cdot$ Rita Cranen $^{3}$}

${ }^{1}$ Klinik für Akut- und Notfallmedizin, St.-Antonius-Hospital gGmbH, Akademisches Lehrkrankenhaus der RWTH Aachen, Eschweiler, Deutschland

${ }^{2}$ Institut und Poliklinik für Arbeits-, Sozial- und Umweltmedizin, Stabsstelle Betriebsärztlicher Dienst, Ludwig-Maximilians-Universitätsklinikum, München, Deutschland

${ }^{3}$ Betriebsärztlicher Dienst, St.-Antonius-Hospital Eschweiler, Eschweiler, Deutschland

\title{
Möglichkeiten der Beschäftigung von schwangeren Mitarbeiterinnen im Gesundheitssystem in Zusammenhang mit SARS-CoV-2
}

Infektionsepidemiologisch zeigen sich aktuell eine Stabilisierung der Neuinfektionsrate und ein Rückgang der akutmedizinischen COVID(Corona Virus Disease)-Patienten. Dennoch wird der tägliche Umgang mit SARS-CoV-2(severe acute respiratory syndrome coronavirus 2)-bestätigten und Verdachtsfällen die Klinik bzw. die Mitarbeiterinnen und Mitarbeiter des Gesundheitswesens zukünftig noch weiter beschäftigen. In einer kürzlich publizierten Mitarbeiterstudie des Universitätsklinikums Münster (UKM) stellten sich Mitarbeiter mit Verdacht auf eine SARS-CoV-2-Exposition gemäß der gültigen Falldefinition des Robert Koch-Institutes (RKI) entweder selbstständig bei der Arbeitsmedizin vor oder wurden nach Exposition zu einem SARS-CoV-2-Kranken im Rahmen der Kontaktverfolgung durch die Krankenhaushygiene ermittelt [1]. Während des Untersuchungszeitraums (vom 11.03.2020 bis 17.04.2020) wurden bei insgesamt 957 Mitarbeiter (insgesamt 11.000 [ca. 1250 ärztliche] Mitarbeiter) 1054 Abstriche auf SARS-CoV-2 vorgenommen. Insgesamt wurden 52 Mitarbeiter $(5,4 \%)$ positiv getestet, von denen $33(63,5 \%)$ in einem Bereich mit direktem Patientenkontakt eingesetzt waren. Von allen positiv getesteten Mitarbeitern hatten 39 Kontakt zu einer SARS-CoV-2positiv getesteten Person, hiervon $21 \mathrm{mit}$ beruflichem Kontakt zu Patienten $(n=1)$ oder anderen Mitarbeitern $(n=20)$. Die Studie verdeutlicht, dass die Ansteckung sowohl über direkten Patientenkontakt, als auch über Mitarbeiter untereinander erfolgen kann. Ein striktes Einhalten der Hygienevorschriften sowie das konsequente Tragen eines Mund-NasenSchutzes am Arbeitsplatz bei jeglichem Kontakt zu Patienten und/oder Mitarbeitern und ein verantwortungsvolles Verhalten von Mitarbeitern bei privaten Kontakten untereinander sind für einen erfolgreichen Infektionsschutz unerlässlich. Der Arbeitgeber sollte bei seinen Unterweisungen regelmäßig konkret darauf hinweisen, insbesondere vor dem Hintergrund, dass die Lockerungen der Beschränkungen im allgemeinen Leben wieder zu häufigeren Kontakten führen.

Basierend auf der aktuell stabilen COVID-Datenlage ist das Bestreben vieler Kliniken, Schwangere aus dem Tele-/ Homeoffice oder aus anderen Bereichen zurück- bzw. herauszuholen, um diese wieder in die primäre Patientenversorgung einzusetzen. Ob eine Übertragung des Virus auf das ungeborene Kind möglich ist, war bis dato unbekannt. In einer retrospektiven Studie konnte in 2 von 22 Neugeborenen, die von COVID19-Müttern geboren wurden, SARSCoV-2-RNA nachgewiesen werden [2]. Die Autoren Zimmermann und Curtis
[3] beschreiben anhand von 9 Fallserien und 2 Fallberichten die Ergebnisse der mütterlichen SARS-CoV-2-Infektion während der Schwangerschaft bei 65 Frauen und 67 Neugeborenen. Zwei Mütter (3\%) wurden intensivpflichtig, bei $30 \%$ der Schwangerschaften traten geburtshilfliche Notfälle auf und $37 \%$ erlitten eine Frühgeburt. $\mathrm{Zu}$ den neonatologischen Komplikationen gehörten das Atemnotsyndrom oder Pneumonie $(18 \%)$, die disseminierte intravasale Koagulopathie (3\%), die Asphyxie (2\%) und 2 perinatale Todesfälle. Vier Neugeborene ( 3 mit Pneumonie) waren trotz strenger Hygienevorschriften während der Geburt und der Trennung von Mutter und Neugeborenem SARS-CoV-2positiv. Diese jüngsten Beobachtungen unterstützen die Möglichkeit einer vertikalen Übertragung von SARS-CoV-2 auf das Kind, sodass Schwangere insbesondere im Rahmen der Coronapandemie geschützt werden sollten.

Studien zu stillenden Müttern existieren bisher nicht. Lediglich in einer Studie war eine von 3 Muttermilchproben durch einen viralen Nukleinsäuretest positiv [4]. Die Frage nach der Möglichkeit einer Übertragung via Muttermilch kann derzeit nicht beantwortet werden. Ebenfalls ist unklar, ob das Stillen das Virus von zuvor infizierten und genesenen Müttern auf ihre Neugeborenen überträgt. Aktu- 
elle Ergebnisse deuten darauf hin, dass das Stillen praktiziert werden kann, nachdem eine Quarantäne abgeschlossen ist und Wiederholungstests negativ sind [5].

Im Rahmen der Gefährdungsbeurteilung gemäß Arbeitsschutzgesetz durch den Arbeitgeber ist immer der Mutterschutz zu berücksichtigen. Gemäß Mutterschutzgesetz muss der Arbeitgeber eine unverantwortbare Gefährdung für die Schwangere ausschließen. Eine Weiterbeschäftigung einer Schwangeren sollte nur in Erwägung gezogen werden, wenn das Infektionsrisiko am Arbeitsplatz nicht höher ist als das der Allgemeinbevölkerung. Diese Maßnahme sollte daher nach individueller Gefährdungsbeurteilung mit genauer Beschreibung der Patientenklientel und Definition der auszuübenden Tätigkeiten unter Einhaltung folgender Punkte erfolgen:

- keine Tätigkeiten mit erhöhten Personen- oder Publikumsverkehr (z. B.

Ambulanzen, Funktionsabteilungen);

- ausschließlich Umgang mit elektiven Patienten, die vor der stationären Aufnahme negativ auf SARS-CoV-2 gescreent wurde;

- keine Tätigkeiten mit engem Körperkontakt zu den Patienten (z.B. akutmedizinische Tätigkeiten, wie z. B. Absaugung der Atemwege, sowie Logopädie oder Physiotherapie);

- die Übertragung von Infektionserkrankungen kann durch Arbeitsschutz bzw. vorhandenen Immunschutz sicher verhindert werden;

- es ist sichergestellt, dass bei Notfällen andere Kollegen übernehmen;

- die Schwangere ist in den Tätigkeiten geübt und fühlt sich sicher.

Die Entscheidung über die Beschäftigungsmöglichkeiten für eine schwangere Mitarbeiterin ist daher eine individuelle Einzelfallentscheidung, die vom Arbeitgeber in Kenntnis des konkreten Arbeitsplatzes in Zusammenarbeit mit der schwangeren Mitarbeiterin unter Beteiligung der Betriebsärztin/des Betriebsarztes getroffen werden sollte $[6$, 7].

\section{Korrespondenzadresse}

Prof. Dr. med. Guido Michels

Klinik für Akut- und Notfallmedizin, St.Antonius-Hospital gGmbH, Akademisches Lehrkrankenhaus der RWTH Aachen Dechant-Deckers-Str. 8, 52249 Eschweiler, Deutschland

guido.michels@sah-eschweiler.de

\section{Einhaltung ethischer Richtlinien}

Interessenkonflikt. G. Michels, U. Ochmann und R. Cranen geben an, dass kein Interessenkonflikt besteht.

Für diesen Beitrag wurden von den Autoren keine Studien an Menschen oder Tieren durchgeführt. Für die aufgeführten Studien gelten die jeweils dort angegebenen ethischen Richtlinien.

\section{Literatur}

1. Schwierzeck C, Correa-Martinez CL, Schneider KN Mellmann A, Hennies MT, Hafezi W, Czeschinski P (2020) SARS-CoV-2 in the employees of a large university hospital. Dtsch Arztebl Int 117:344-345

2. Patanè $L$, Morotti $D$, Giunta MR, Sigismondi $C$, Piccoli MG, Frigerio L, Mangili G, Arosio M, Cornolti G (2020) Vertical transmission of COVID19: SARS-CoV-2 RNA on the fetal side of the placenta in pregnancies with COVID-19 positive mothers and neonates at birth. Am J Obstet Gynecol. https://doi.org/10.1016/j.ajogmf.2020. 100145

3. Zimmermann P, Curtis N (2020) COVID-19 in children, pregnancy and neonates: a review of epidemiologic and clinical features. Pediatr Infect Dis J39(6):469-477

4. Wu Y, Liu C, Dong L, Zhang C, Chen Y, Liu J, Zhang C, Duan C, Zhang H, Mol BW, Dennis CL, Yin T, Yang J, Huang H (2020) Coronavirus disease 2019 among pregnant Chinese women: case series data on the safety of vaginal birth and breastfeeding. BJOG. https://doi.org/10.1111/1471-0528.16276

5. Lang GJ, Zhao H (2020) Can SARS-CoV-2-infected women breastfeed after viral clearance? JZhejiang Univ Sci B 21(5):405-407

6. Bayerisches Staatsministerium für Familie, Arbeit und Soziales (2020) Informationen zum Mutterschutz im Zusammenhang mit dem Coronavirus SARS-CoV-2/COVID-19 - Stand 8. Mai 2020. https://www.stmas.bayern.de/ imperia/md/content/stmas/stmas inet/200508 corona_info_mutterschutz_final.pdf. Zugegriffen:02.06.2020

7. Ministeriums für Arbeit, Gesundheit und Soziales des Landes Nordrhein-Westfalen (2020) Arbeitsmedizinische Empfehlungen zur Beschäftigung von schwangeren und stillenden Frauen im Zusammenhang mit dem Coronavirus SARS-CoV-2/COVID-19-Erkrankung. https:// www.brd.nrw.de/corona/pdf_corona/200424 Arbeitsmedizinische-Einschaetzung.pdf. Zugegriffen:02.06.2020
Med Klin Intensivmed Notfmed 2020 - 115: 486-487

https://doi.org/10.1007/s00063-020-00704-0

(c) Springer Medizin Verlag $\mathrm{GmbH}$, ein Teil von Springer Nature 2020

G. Michels · U. Ochmann · R. Cranen

Möglichkeiten der

Beschäftigung von

schwangeren

Mitarbeiterinnen im

Gesundheitssystem in Zusammenhang mit SARSCoV-2

\section{Zusammenfassung}

Eine Weiterbeschäftigung von Schwangeren in Zusammenhang mit COVID (Corona Virus Disease) sollte nur in Erwägung gezogen werden, wenn das Infektionsrisiko am Arbeitsplatz nicht höher ist als das der Allgemeinbevölkerung. Diese Maßnahme sollte daher nach individueller Gefährdungsbeurteilung mit genauer Beschreibung von Patientenklientel und Definition der auszuübenden Tätigkeiten erfolgen.

\section{Schlüsselwörter}

Schwangerschaft · Mitarbeiter · Prävention · Coronavirus · Akutmedizin

\section{Possibilities of employment of} pregnant women in the health care system in association with SARS-CoV-2

\section{Abstract}

Continued employment of pregnant women related to COVID (Corona Virus Disease) should only be considered if the risk of infection in the workplace is not higher than that of the general population. This option should therefore be carried out after an individual risk assessment with a precise description of the patient clientele and definition of the activities to be performed.

Keywords

Pregnancy - Employees - Prevention

Coronavirus · Acute care medicine 